\title{
Survey on Some Scene Based Nonuniformity Correction Algorithms for Infrared Images
}

\author{
Shweta Wanmali ${ }^{1}$, Prof. Rajesh Shekokar ${ }^{2}$ \\ Student, Electronics and Telecommunication, RMD Sinhgad College of Engineering, Pune, India ${ }^{1}$ \\ Assistant Professor, Electronics and Telecommunication, RMD Sinhgad College of Engineering, Pune, India ${ }^{2}$
}

\begin{abstract}
In this paper, scene based nonuniformity correction algorithms for the infrared images are discussed. The quality of infrared images gets affected due to spatial fixed pattern noise in IRFPA, and it is mainly caused by nonuniformity of the detectors' pixel to pixel variation. For the infrared focal plane arrays image uniformity is necessary. To correct this nonuniformity, various methods are presented in recent years. Some of them i.e. NN-NUC, CS-NUC, IR-NUC and GE-NUC are discussed here. We concentrate on the main contributions of the methods and their advantages and disadvantages. Also we compare these methods to distinguish the better method to eliminate ghost artifacts.
\end{abstract}

Keywords: IRFPA, Nonuniformity, Nonuniformity correction methods, Scene based nonuniformity correction algorithms, Elimination of ghost artifacts.

\section{INTRODUCTION}

The infrared focal plane arrays is rapidly developing as the use of infrared images is widely increasing in military, industrial, medical, and other fields. An IRFPA comprises of a variety of free infrared detectors put at the focal plane of the imaging system. Tragically, every detector in the array can perform not the same as each other. It has different response levels in the same stimulus. This misadjustment between the detectors response leads to the presence of some noise in the resultant images. When NUC is operated on the scene it affects the sensitivity of device and decrease the performance of infrared cameras. Also there may appear some artifacts. Ghosting is a guarantee impact due to the NUC worked on the scene. To solve this problem; there are two main categories of nonuniformity correction (NUC) methods are developed i.e. Calibration based (CB) method and Scene based (SB) method [1].

In the real time imaging system calibration based methods are unsuitable because of their pre-processing property, also their correction coefficients are prestored but detectors response changes with time. After some time of operation the correction parameters of these methods are not applicable for longer time period. Due to this IR image's performance decreased. By using motion related features in image sequences the true infrared images form the fixed pattern noise which is identified by the scene based techniques [2]. Hence to get the better performance some methods are presented in recent years. In this paper some of them i.e. NN-NUC, CS-NUC, IR-NUC and GE-NUC are described shortly.

The paper divided into four sections $2^{\text {nd }}$ section contains detail information of the respected scene based nonuniformity correction methods and $3^{\text {rd }}$ section contains comparison of the methods. To compare these methods we refer an example from [6] to visualize the changes found in the images. And we can verify the better method for denoising.

\section{SCENE BASED METHODS}

In this section the brief detail of some scene based nonuniformity correction methods is presented. We cover the main contribution of the method and their advantages and disadvantages. The methods which taken here are, Nural Network based nonuniformity correction, Constant Statistic constraint based nonuniformity correction, Interframe Registration based nonuniformity correction and Ghost Eliminating nonuniformity correction method. These methods' basic function is to minimize ghosting artifacts which comes due to spatial fixed pattern noise in infrared images. We have referred NN-NUC from [3], CS-NUC from [4], IR-NUC from [5], and GE-NUC from [6]

\section{A. NN-NUC}

This method uses new adaptive learning rate rule into the rough calculation of gain and offset of each detector. This learning rate is choosing for it will depend on the spatial variation of the read-out data of one frame, and also it will depend on the temporal motion between two successive frames. This adjustment allow the detectors input data get fits in assumption and also follow the temporal variations in the operation point of each detector. Furthermore, the neurons parameters calculation is improved by considering the error function of the updated detectors' parameters which are 


\section{ISO 3297:2007 Certified}

Vol. 5, Issue 4, April 2017

depends on the retinomorphic assumption. In the paper[3] a new method is proposed, in which first they employs local scene statistics to remove low frequency noise and ghosting artifacts, and then uses improved neural network algorithms to filter out high frequency non-uniformity noise from IRFPA.

In neural network algorithm the main improvement is focused on two parts: 1) infrared image data are normalized, 2) self adaptive weighted mean filter of five-adjective pixels is designed.

The advantage of this method is; it increase the ability of the scene based method to estimate the nonuniformity parameters and it also increase the methods capability to overcome the ghosting artifacts.

\section{B. CS-NUC}

This method is used for correcting gain and offset errors without special calibration images and procedures. During normal operation of the sensors the natural constraints which are available as natural constraints must be utilized by a scene based nonuniformity calibration method. The nonuniformity correction algorithm's constraints are roughly constant for each pixel. This constant statistics method assumes that for all pixels the temporal mean and variances are identical. This constratints assumes that the photoarray is periodically moving. And also the average statistics each pixel should constant over a long time period.

In this method to remove ghost artifacts the changes in each pixel detects and compare them with threshold value mean and mean deviation which estimates only update if the change is greater than the threshold. This mechanism disallows biased estimates from stationary objects in scenes and helped to sample data better. Choosing proper threshold is important for getting the desired performance. Very low threshold value cannot properly remove the ghost artifacts. And also too high threshold value can cause too few samples for estimation of mean and mean deviation. In this method proper range of threshold value (10\%to $25 \%$ full scale) is found.

In CS-NUC method the simple deghosting module compares the magnitude of change at each pixel with fixed threshold value to reduce ghost artifacts. The mean and mean deviation values update only when the change is greater than the threshold. This mechanism prevents biased mean and mean deviation values from stationary objects in scenes and it help to sample the data better. The similar mechanism can be use to implement for any scene-based nonuniformity correction technique.

\section{IR-NUC}

The method calculates detector parameters and by minimizing the mean square error between two registered image frames. Authors introduce a new masked phase correlation algorithm to obtain reliable shift estimates in the presence of fixed pattern noise. To reduce ghost artifacts they focus on interframe registration based least-mean-square-error algorithm (IRLMS) which is developed by Zuo et al. This technique minimizes the mean square error between two properly registered image frames to make same output value with detectors of same scene.

The advantages of this method are fast convergence and minor residual error by improving the IRLMS method. The performance of this method is depends on the accuracy of registration estimates. So the method performed in steps:

1. Improve accuracy of registration under condition of nonuniformity noise

2. Assume the radiation generates from scene does not change during the time between image frames

3. As translational motion between two image frames is taken into consideration, the estimation error may result from scene rotation, warping, etc. These errors can minimize by attenuating these frames' contribution to the updating process.

These outlier values caused by local motion should be excluded to prevent a large degree of influence on NUC parameters. In this method performance and the robustness is improved and here quantitative analysis takes place which showed the method has faster convergence rate than the IRLMS method.

The correction output shows the residual nonuniformity is too low to be detected by the naked eyes, and almost no ghosting artifact can be detected. The strength of the proposed algorithm lies in its reasonably simple assumptions and smaller calculation and memory requirements, which make it more competitive in real-time processing. The correction errors may result from local motion, scene rotation, changes of scene irradiation, etc. When the scene objects are imaged at a relatively small distance, warping of the images should also be taken into account. However, corresponding counter measures are not so complicated in our method.

\section{GE-NUC}

This method shows the adaptive image registration non uniformity correction method with the function of eliminating ghost artifacts. Firstly, With respect to time period continuous image sequence, the displacement vectors based on their row and column projections are calculated then, by bidirectional image registration, the overlapped area matrices of two frames obtained accurately. Also at that time, a variance threshold is set to judge the scene classification, and then, displacement revising added to decrease stripe noise. By using the mean-square-blunder cycle calculation, the pickup 
and balance coefficients of the covered zone can be overlapped continuously with the picture grouping adaptively. The advantages of this calculation are high productivity of picture preparing, low computational many-sided quality. A clean infrared image sequence and two real infrared datasets with non uniformity are used to compare the ability and correction performance of different algorithms. The output of the algorithm gives the precise output than other methods. The advantages of this method are few ghosting artifacts, low computational complexity, and convenient hardware implementation with real time processing.

But there are some limitations may occur in some special situations i.e. when scene is static for long term, the displacement which is calculated can remove the stripe noise but it may reduce image resolution and image may get blur. Again when image sequence is filled with full nonuniformity noise, the image registration will be affected severely and the performance will decrease. Also the algorithm does not consist any function to express input parameters i.e. rotation and nonrigid moving, hence the algorithm is not suitable for these situations.

\section{III.COMPARISON OF METHODS}

In this section, we take comparison of the respected scene based methods. We compare them by referring the results demonstrated in [6]. They compare the results with clean infrared sequence. The data set was collected by using an $256 \times 320 \mathrm{HgCdTe}$ IRFPA camera which is operating in the 8-14 $\mu \mathrm{m}$ range. The images are taken from the NUST campus. The visual parameters i.e. quantity of the ghosting artifacts and edge blur are compared. And also the methods are compared on the basis of computational complexity and feasibility of the respected methods.

\section{A. NN-NUC}
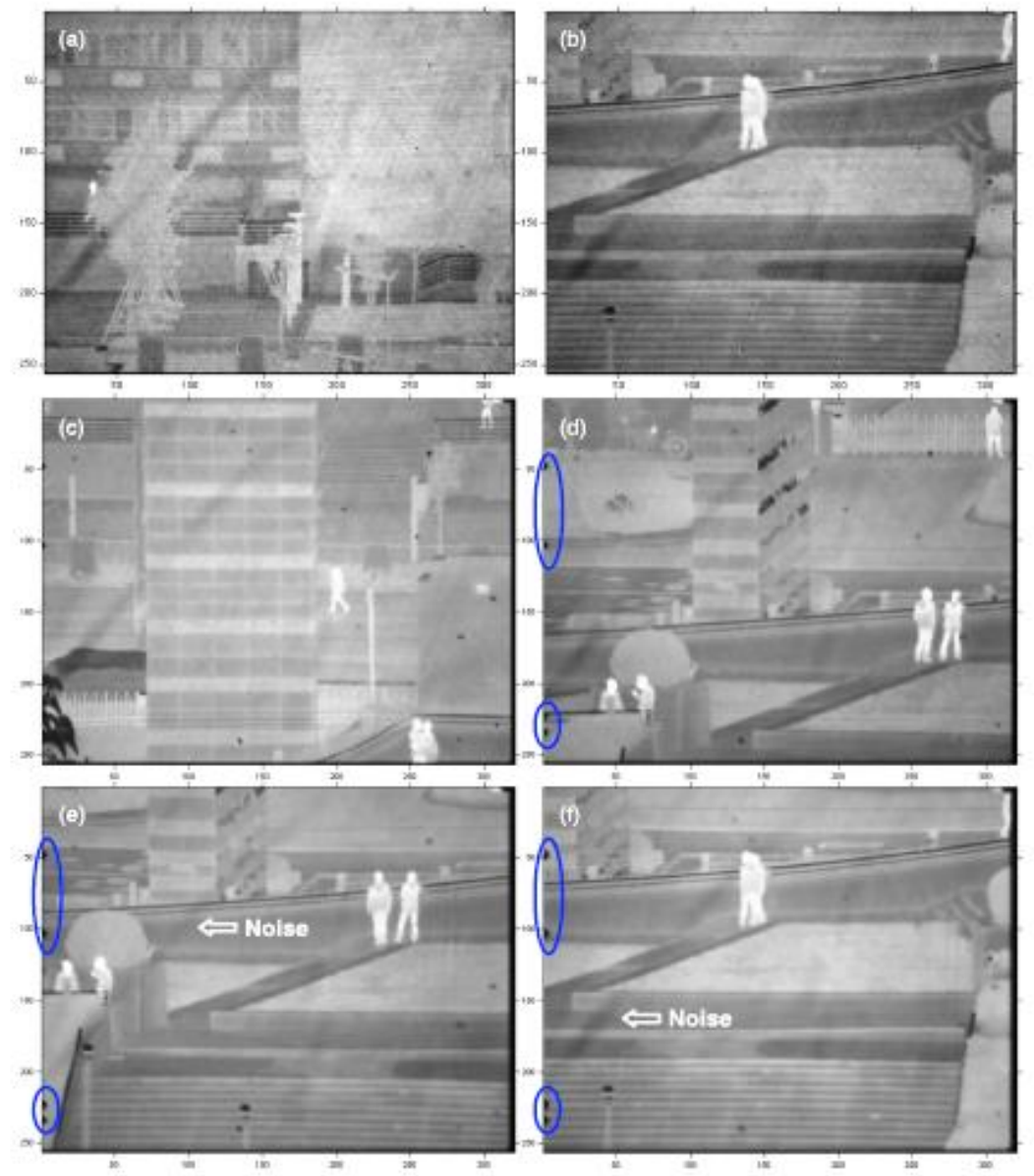

Fig. 1. (a) Frame 1 and (b) frame 200 of the raw infrared image sequence of NUST campus. (Media 1) (c) frame 50, (d) frame 100, (e) frame 150, and (f) frame 200 of the sequence of NN-NUC.

Here fig (a) and (b) are the input images and (c),(d),(e),(f) are the results of the method NN-NUC In this method some stripe noise is observed and also some low spatial frequency noise is observed. Again due to discrete bad pixels some 
dark area appears in frame which is shown by blue circle of frame 150 and frame 200 . We can see no edge blur occur in result images and medium ghost artifacts occurs. This method has low computational complexity as it is a scene based method.

B. CS-NUC
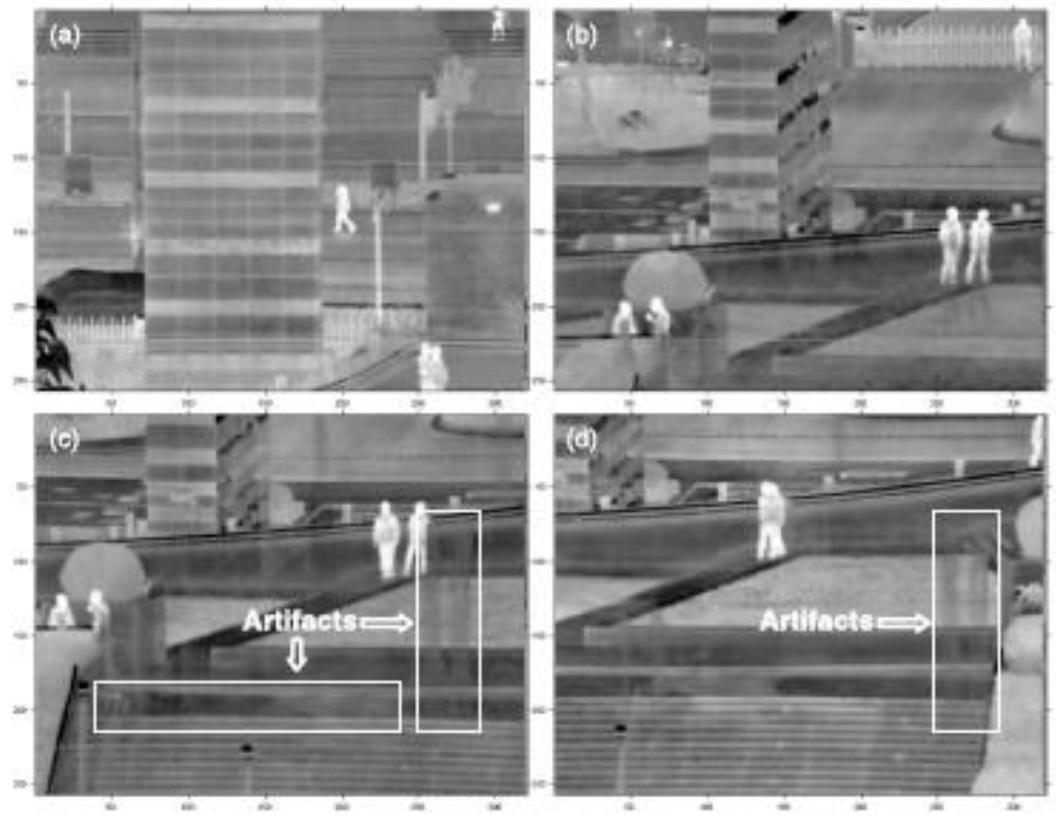

Fig. 2. (Media 2) (a) Frame 50, (b) frame 100, (c) frame 150, and (d) frame 200 of the sequence of CS-NUC.

Here in this figure we can observe the stripe noise is removed. But some artifacts still occur here. We can see in above picture some sculpture at the lower left corner and also there is no edge blur occurs and high ghosting artifacts occurs in results.

C. IR-NUC
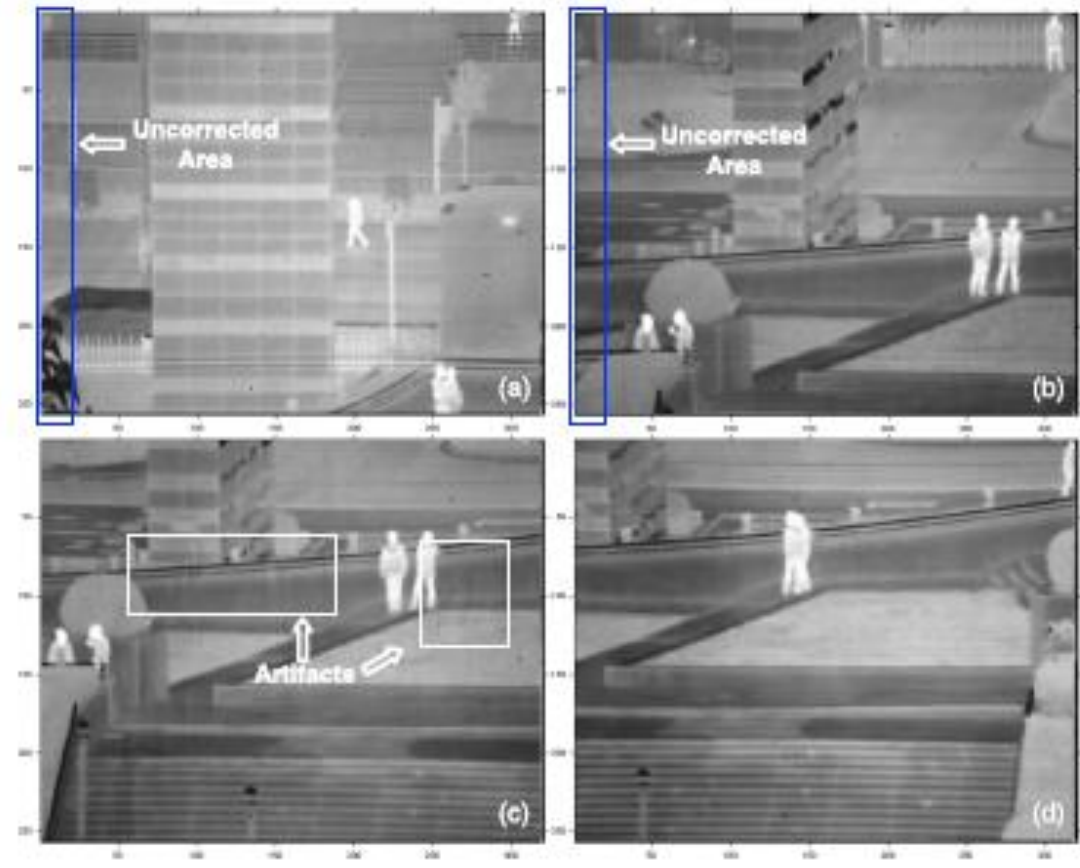

Fig. 3. (Media 3) (a) Frame 50, (b) frame 100, (c) frame 150, and (d) frame 200 of the sequence of IR-NUC. 
This method is more efficient than above two methods. But here also some uncorrected area is found at the left border. This method calculates displacement in horizontal direction so we can find the overlapped area in right part of image.

D. GE-NUC
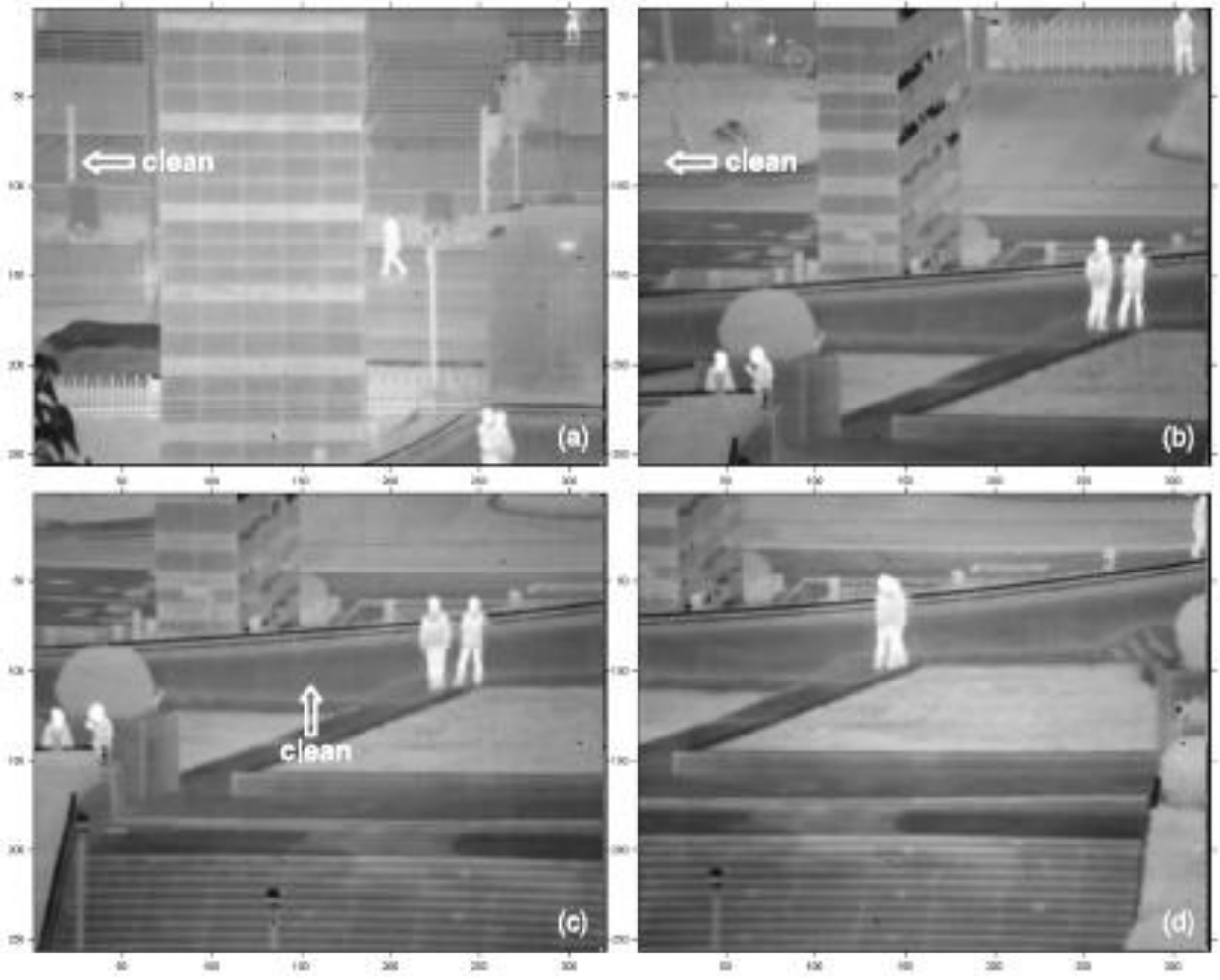

Fig. 4. (Media 4) (a) Frame 50, (b) frame 100, (c) frame 150, and (d) frame 200 of the sequence of GE-NUC.

The above issues observed in these NN-NUC, CS-NUC, IR-NUC methods are removed in GE-NUC method. The images or results of GE-NUC are clear and no any noise is occurs. Also this method's speed is higher than other methods. The summary is given by following table.

Table1. Summary

\begin{tabular}{|l|l|l|}
\hline Method & Ghost Artifacts & Edge Blur \\
\hline NN-NUC & Medium & - \\
\hline CS-NUC & High & - \\
\hline IR-NUC & Medium & High \\
\hline GE-NUC & Low & Low \\
\hline
\end{tabular}

\section{IV.SUMMARY}

This paper has compared different scene based nonuniformity correction algorithms for infrared focal plane arrays which are feasible to eliminate ghosting artifacts. The brief discussion about the main contributions, advantages and disadvantages is taken. The comparison of these methods is discussed to distinguish better method to eliminate ghost artifacts. To the best of our knowledge the ghost eliminating method is better than other methods as this method have less ghosting artifacts, low computational complexity, and convenient hardware implementation with real time processing. 


\section{IJIREEICE

\section{REFERENCES}

[1] D. A. Scribner, M. Kruer, and J. Killiany, "Infrared focal plane array technology," Proc. IEEE, vol. 79, no. 1, pp. 66-85, Jan. 1991.

[2] Q Wang, J M Dai, X G Sun and Y L Wang, "A New Scene-Based Non-Uniformity Correction Algorithm for Infrared Focal Plane Array", Department of Automation Measurement \& Control, Harbin Institute of Technology, Heilongjiang, Harbin, 150001, China

[3] Sergio N. Torres, Cesar San Martin, Daniel G. Sbarbaro, and Jorge E. Pezoa "A Neural Network for Nonuniformity and Ghosting Correction of Infrared Image Sequences", Department of Electrical Engineering, University of Concepci' on, Casilla 160-C, Concepci'on, Chile.

[4] John G. Harris and Yu-Ming Chiang,"Nonuniformity Correction of Infrared Image Sequences Using the Constant-Statistics Constraint", IEEE transactions on image processing, vol. 8, no. 8, august 1999

[5] Zuo, Q. Chen, G.H. Gu, X.B. Sui, “Scene-based nonuniformity correction algorithm based on interframe registration”, J. Opt. Soc. Am. A 28 (2011) 1164-1176.

[6] Junjie Zeng, Xiubao Sui, Hang Gao, "Adaptive Image-Registration-Based Nonuniformity Correction Algorithm With Ghost Artifacts Eliminating for Infrared Focal Plane Arrays”, IEEE Photonics Journal, August 18, 2015

[7] L. Rui, Y. Yin-Tang, Z. Duan, and L. Yue-Jin, "Improved neural network based scene-adaptive nonuniformity correction method for infrared focal plane arrays,” Appl. Opt., vol. 47, no. 24, pp. 4331-4335, Aug. 2008.

[8] R. C. Hardie, F. Baxley, B. Brys, and P. Hytla, "Scene-based nonuniformity correction with reduced ghosting using a gated LMS algorithm," Opt. Exp., vol. 17, no. 17, pp. $14918-14$ 933, Aug. 2009.

[9] Y. Tendero a , J. Gillesb. "ADMIRE: a locally adaptive single-image, non-uniformity correction and denoising algorithm: application to uncooled IR camera"

[10] John G. Harris and Yu-Ming Chiang, "Minimizing the Ghosting Artifact in Scene-Based Nonuniformity Correction”,Proc. SPIE Vol. 3377, p. 106-113, Infrared Imaging Systems: Design, Analysis, Modeling, and Testing IX

\section{BIOGRAPHIES}

Ms. Shweta D. Wanmali is M.E. Student, studying VLSI and Embedded system in RMD Sinhgad college of Engineering, Pune. Author has her personal interest in the domain of Image Processing.

Prof. Rajesh U. Shekokar is working as a Assistant Professor in Department of E\&TC. His area of interest in the domain of VLSI and Embedded System. 